\title{
Dimorfismo sexual y morfología funcional de las extremidades de Loxosceles laeta (Nicolet, 1849)
}

\section{Sexual size dimorphism and functional morphology of legs of Loxosceles laeta (Nicolet, 1849)}

\author{
Mauricio Canals ${ }^{1}$, Andrés Taucare-Rios ${ }^{2}$, Rigoberto Solis $^{3}$ \& Lucila Moreno ${ }^{4}$ \\ ${ }^{1}$ Departamento de Medicina y Programa de Salud ambiental, Escuela de Salud Pública, Facultad de Medicina, Universidad \\ de Chile. E-mail: mcanals@uchile.cl \\ 2Departamento de Ciencias Ecológicas, Facultad de Ciencias, Universidad de Chile. \\ ${ }^{3}$ Departamento de Ciencias Biológicas Animales. Facultad de Ciencias Veterinarias y Pecuarias. Universidad de Chile. \\ ${ }^{4}$ Departamento de Zoología. Facultad de Ciencias Naturales y Oceanográficas. Universidad de Concepción. \\ Corresponding author: Mauricio Canals. Departamento de Medicina y Programa de Salud ambiental, Escuela de Salud \\ Pública, Facultad de Medicina, Universidad de Chile. E-mail: mcanals@uchile.cl
}

\begin{abstract}
RESUMEN
Las relaciones alométricas entre rasgos morfológicos aportan evidencia de las fuerzas evolutivas que actúan sobre un rasgo particular. Las arañas que viven a ras de suelo son menos dimórficas que las que construyen telas, lo que ha sido atribuido a sus diferentes estrategias reproductivas y de forrajeo. Se ha propuesto que las largas extremidades en machos con respecto a la masa conducen a una optimización de los costos de locomoción. El objetivo de este estudio fué caracterizar la variabilidad de las dimensiones de las extremidades en la araña del rincón Loxosceles laeta y comparar las relaciones alométricas de las extremidades locomotoras entre ambos sexos. Tanto machos como hembras mostraron una alometría negativa de las extremidades, es decir las arañas con tamaño mayor tienen patas proporcionalmente más cortas que aquellas con cefalotórax más cortos, lo cual puede ser explicado por causas biomecánicas. Los machos presentaron patas más largas que las hembras tanto en tamaño absoluto como relativo. Esto sugiere que los machos tienen una mayor eficiencia locomotora. El tamaño corporal pequeño está relacionado con bajo costo de transporte lo que asociado con patas largas es la morfología esperada para los machos más activos y móviles en esta especie.
\end{abstract}

Palabras Clave: Loxosceles, Dimorfismo sexual, Alometría, Extremidades.

\begin{abstract}
Alometric relationships among morphological characters provide evidence of the evolutionary forces that act on a particular trait. Spiders that live on the ground are less dimorphic than orb web spiders, which have been attributed to their different reproductive and foraging strategies. It has been proposed that long legs with respecto to the body size in males, yields an optimization of locomotion costs. The aim of this study was to characterize the variability of the length of legs in the Chilean recluse spider Loxosceles laeta and to compare the alometric relationships of the locomotor legs in males and females. Both sexes showed negative alometric relationships between the length of the legs and the lengtgh of the cephalotorax, ie spiders of large body size have proportionally shorter legs than that of small body size, which may be explained by bio mechanic causes. The males showed longer legs than females both in absolute and relative terms. That means that males have a higher locomotor efficiency than females. The relative small body size is related to a low transportation cost, which associated with long legs is the expected morphology for the more active and mobile males in this species.
\end{abstract}

KEYwords: Loxosceles, Sexual dimorphism, Alometry, Legs.

\section{INTRODUCCION}

La anatomía funcional establece que el dimorfismo sexual no solo se debe a diferencias en masas sino que también a diferencias en el tamaño relativo de las diferentes partes del cuerpo de un organismo (Thompson 1961). Las relaciones alométricas entre rasgos morfológicos aportan evidencia de las fuerzas evolutivas como selección sexual, que actúan sobre un rasgo particular (Anderson 1994; Stern \& Emlen 1999; Emlen 2008). 
En arañas hay evidencia que la selección que favorece la fecundidad se asocia a un tamaño corporal mayor con el consiguiente menor tamaño relativo de las patas (Head 1995; Foellmer \& Moya-Larraño 2007; Santos 2007). Otras hipótesis proponen la selección de tamaños corporales pequeños en machos asociada a relajación de la selección por tamaños grandes en bajas densidades poblacionales (hipótesis de Ghiselin-Reiss) (Ghiseling 1974; Reiss 1989); o también asociada a evitar el canibalismo; o a una mayor velocidad para acceder a la hembra (Elgar \& Fahey 1996); o a una maduración precoz de los machos (Vollrath \& Parker 1992).

Se ha reportado una gran diferencia en tamaño corporal y en la longitud de las patas en arañas de telas orbitales que viven al interior vegetación, con machos pequeños con patas largas (Foellmer \& Moya-Larraño 2007), generando la hipótesis de gravedad que propone que arañas macho de pequeño tamaño tienen mayor velocidad para alcanzar a las hembras "escalando" contra la gravedad (ver objeciones de Prenter et al. 2010a, 2010b; Morse 2014), incrementando la probabilidad de apareamiento (Anderson 1994; Fairbairn 1997, 2013; Eberhard et al. 2000). Esto podría ser mediado por selección natural o sexual por tamaño grande de la hembra, tamaño pequeño del macho o ambas (Ghiselin 1974; Vollrath \& Parker 1992; Eberhard 2004; Kruger 2005; Sánchez-Quiróz et al. 2012). Se ha propuesto que las diferencias sexuales en las estructuras locomotoras están asociadas a la conducta más activa de los machos (Thornhill \& Alcock 1983; Gasnier et al. 2002). Sin embargo otros autores proponen que la elongación de las patas puede no estar asociada solamente con locomoción (Elgar et al. 1990; Peters \& Campbell 1991; Eberhard \& Marin 1996; Tseng \& Rowe 1999; Ohtsuka \& Huys 2001; Malmgren \& Thollesson 2001; Framenau 2005). Recientemente Grossi \& Canals (2015) revisaron el rol de la energía en el dimorfismo sexual de las arañas incluyendo aquellas arañas que no necesariamente viven en lo alto del follaje, proponiendo que el tamaño corporal pequeño y las patas largas corresponden a un diseño de bajo costo energético (Grossi \& Canals 2015) con un incremento en la eficiencia locomotora.

Las arañas que viven a ras de suelo son menos dimórficas que las que construyen telas, lo que ha sido atribuido a sus diferentes estrategias reproductivas y de forrajeo (Enders 1976; Prenter et al. 1997, 1998, 1999). A pesar de esto hay evidencias de dimorfismo sexual en las estructuras locomotoras en estas arañas (e.g., Gasnier et al. 2002; Framenau 2005). Montgomery (1910) reportó que los machos tienen patas relativamente mayores que las hembras, sugiriendo que es el resultado de un comportamiento nómade de los machos cuando alcanzan la madurez sexual. Esta idea es soportada por estudios de la actividad locomotora de las arañas lobo (Lycosidae), en las cuales los machos son el sexo más activo (Hallander 1967; Richter et al. 1971; Framenau et al. 1996; Framenau 2005). También en arañas migalomorfas se ha reportado dimorfismo sexual con machos más pequeños pero con patas más largas (Calderon et al. 1990; Foelix 2011; Santos 2007; Grossi et al. 2016a) y recientemente en un estudio alométrico se ha propuesto que en la gran araña Grammostola rosea (Walckenaer, 1837) las largas extremidades en machos con respecto a la masa conducen a una optimización de los costos de locomoción (Grossi et al. 2016b).

La araña del rincón, L. laeta, es una araña errante, solitaria, asociada al ambiente doméstico, cuyos habitats preferidos son los rincones obscuros dentro de closet, entretechos, estantes entre ropas y toallas, o detrás de cuadros. También puede ser encontrada en los exteriores bajo rocas, troncos, grietas, etc. Construyen pequeñas telas irregulares, donde habitualmente dejan su ooteca. Su descripción original se debe a Nicolet (1849), sin embargo llama la atención que en la descripción original de Nicolet la fórmula de patas de L. laeta es 4-1-2-3 y para L. rufipes es 4-2-1-3, mientras que para Lucas (1834) L. rufipes tiene fórmula 4-1-2-3. Posteriormente Simon (1907) revisó el material de Loxosceles sudamericanas consideró sinónimos L. laeta, L. rufipes y L. nigella, sin hacer caso a la diferencia de patas, conservando sólo el nombre de L. laeta. Gertsch en 1958 redescribió a $L$. laeta con la fórmula 4-2-1-3, lo que ha sido refrendado por Platnick (2004). Se distribuye en Chile, Perú, Ecuador, Argentina y Brasil. Introducida en Estados Unidos, Canadá, Australia y Finlandia (Gertsch \& Ennik 1983). Su color es pardo claro u oscuro, dependiendo del estado de desarrollo y del sexo. Los estados inmaduros y los machos son más claros. La hembra adulta mide alrededor de 1 a 1,5 $\mathrm{cm}$ en su cuerpo, pudiendo llegar a $4,5 \mathrm{~cm}$ con las patas extendidas (Schenone 2003). Fuera de la descripción de $L$. coquimbo (Gertsch, 1967) y el hallazgo reciente de L. surca (Gertsch, 1967) en el Norte de Chile, todos los ejemplares son atribuidos a L. laeta (Alfaro et al. 2013; Galiano 1967; Canals et al. 2004, 2013, 2015; Canals \& Solís 2013, 2014; Platnick 2004; Taucare-Ríos 2011).

En L. laeta existe variabilidad individual y dimorfismo sexual, por ejemplo la fórmula de patas aceptada es 4-2-1-3 pero ocasionalmente, se ha reportado que las hembras tienen las dos primeras patas de igual magnitud (Gertsch 1967) y algunos machos tienen la segunda pata mayor que la cuarta dando la fórmula 2-4-1-3 (Gertsch 1967; Gertsch \& Ennik 1983). Siendo una araña errante y aunque se ha reportado el dimorfismo sexual de $L$. laeta, no existen estudios alométricos de los órganos locomotores en esta especie. El objetivo de este estudio es caracterizar la variabilidad de las dimensiones de las extremidades en esta especie y comparar las relaciones alométricas de las extremidades locomotoras entre ambos sexos. 


\section{MATERIAL Y MÉTODOS}

Se examinó el material de $L$. laeta del territorio continental de Chile del Museo Nacional de Historia Natural, del Museo de Zoología de la Universidad de Concepción y del Museo de Zoología de la Universidad de La Serena. Los datos fueron filtrados de acuerdo a los siguientes criterios: (i) los datos debían tener información con la identificación del ejemplar y la localidad, ii) se incluyeron después del examen de los ejemplares minimizando errores, iii) los datos debían estar asociados al nombre del zoólogo que determinó la especie para controlar problemas taxonómicos y iv) el ejemplar debía ser un adulto y estar completo. Se agregaron además 5 individuos capturados en La Pintana. Después de filtrar los datos se obtuvieron 42 individuos, 27 hembras y 15 machos.

Todos los individuos fueron examinados con lupa observando los caracteres taxonómicos propios de la especie. Sólo se realizaron disecciones en los individuos de La Pintana. Se fotografiaron con cámara digital (NIKON D70, Japón) los individuos junto a una referencia de dimensión conocida que podía ser una reglilla graduada $(10 / 7 \mathrm{~mm})$ o una aguja medida con caliper electrónico de Vernier. A cada individuo se le tomó un número variable de fotografías, suficiente para mostrar cada artejo de cada una de las patas. Las fotografías fueron analizadas con software morfométrico (ImageJ $1.47 \mathrm{~b}$ software $\left.{ }^{\circledR}\right)$. Se midieron individualmente todos los artejos: tarso, metatarso, tibia, patela y fémur de cada pata y la longitud del cefalotórax. Se consideró la longitud de cada pata como la suma de las longitudes de cada artejo (Gertsch \& Ennik 1983). En cada artejo la medida se realizó desde el punto medio del extremo proximal hasta el punto medio del extremo distal.

Los datos fueron analizados con el software estadístico STATA 11.0. Se realizó primero estadística descriptiva de los datos. Se compararon las dimensiones de las patas con análisis de la covarianza de dos vías, considerando la longitud de las patas $\left(\mathrm{P}_{\mathrm{i}}\right)$ como variable respuesta, el sexo ( H o M) y el tipo de pata (1-4) como variables independientes y la longitud del cefalotórax (CT) como covariable. El nivel de significación fue $\alpha=0.05$. Se realizaron regresiones potenciales $\left(\mathrm{P}_{\mathrm{i}}=\mathrm{aCT}^{\beta}\right.$ o equivalentemente $\log \left(\mathrm{P}_{\mathrm{i}}\right)=\log (\mathrm{a})$ $+\beta \log (\mathrm{CT}))$ estableciendo asi las relaciones alométricas para cada pata y sexo, y un análisis de covarianza para estudiar la homogeneidad de las pendientes.

Además se consideraron las medidas relativas de cada pata $\left(\operatorname{Pr}_{1}-\operatorname{Pr}_{4}\right)$, donde $\operatorname{Pr}_{\mathrm{i}}=\mathrm{P}_{\mathrm{i}} / \mathrm{CT}$, indepéndizandolas del tamaño corporal y se realizó un análisis de componentes principales determinando las raíces que explican la variabilidad de los datos, análisis multivariado de la varianza entre los sexos (MANOVA) con el objetivo de buscar respuestas diferenciales correlacionadas y un análisis discriminante determinando las variables locomotoras que explican el dimorfismo en esta especie.

\section{RESULTADOS}

Todos los ejemplares revisados se ajustaron adecuadamente a las descripciones a las de $L$. laeta realizadas por Gertsch (1967) y Gertsch \& Ennik (1983). La relación entre el ancho y largo de la tibia del palpo de los machos fue $0,345 \pm 0,029$.

Los machos mostraron mayores longitudes de patas que las hembras $\left(\mathrm{F}_{1,159}=86,52, \mathrm{p}<<0,001\right)$ y existieron diferencias en las longitudes entre las patas $\left(\mathrm{F}_{3,159}=18,06, \mathrm{p}<<0,001\right)$, sin interacción entre el tamaño de las patas y el sexo (Tabla 1). Las comparaciones múltiples muestran que las patas 1 y 3 no son diferentes $(p=0,33)$, pero el resto de las patas difieren entre sí $(p<0,05)$ (Fig. 1). La fórmula de patas es igual en ambos sexos: 4-2-1-3.
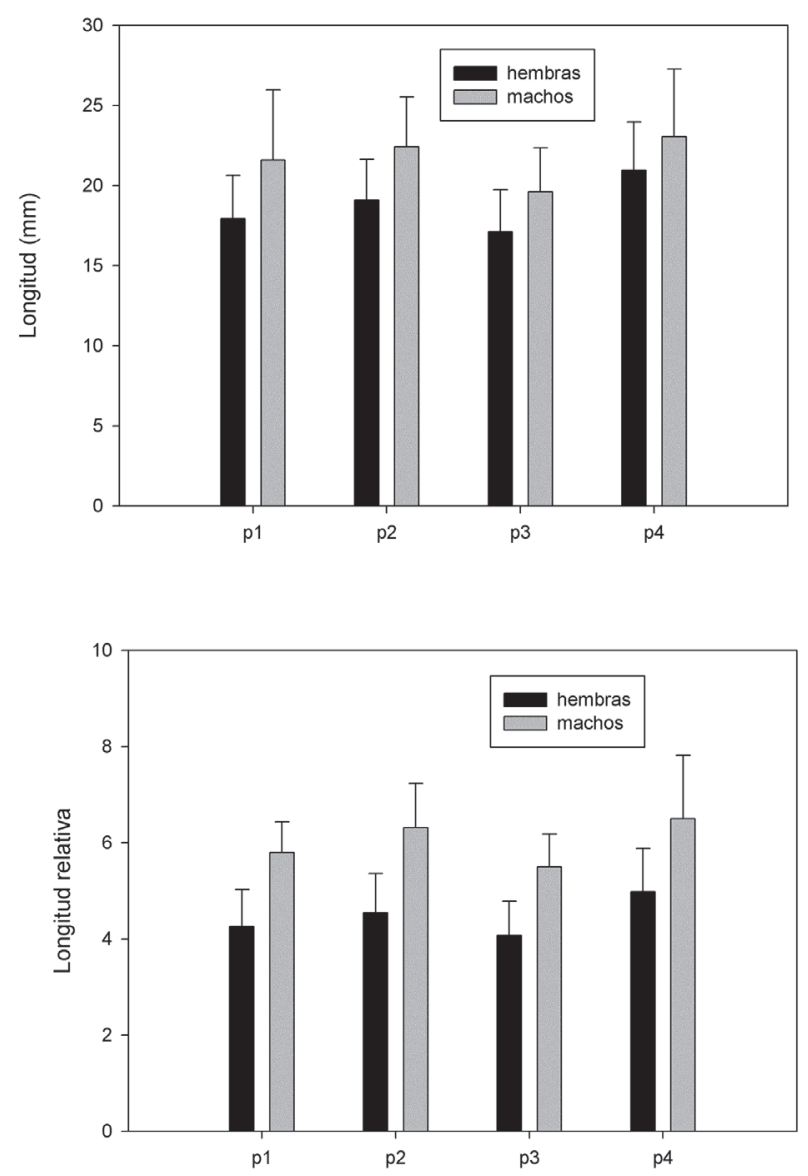

Figura 1. Dimensiones de las extremidades de L. laeta. Dimensiones absolutas (arriba) y relativas (abajo) de las extremidades locomotoras de hembras y machos de L. laeta.

FIgURE 1. Absolute (up) and relative (down) lengths of locomotor legs of females and males of L. laeta. 
Existió una clara relación entre la longitud de las patas y la longitud del cefalotórax $\left(\mathrm{F}_{1,152}=26,62, \mathrm{p}<<0,001\right)$, sin diferencias entre las pendientes entre $\operatorname{sexos}\left(\mathrm{F}_{1,152}=0,42, \mathrm{p}=\right.$ $0,52)$ (Fig. 2) ni entre patas $\left(\mathrm{F}_{3,152}=0,36, \mathrm{p}=0,78\right)$.

El análisis multivariado confirmó las diferencias en la dimensión de las patas entre machos y hembras $(\lambda$-Wilk $=0,47, \mathrm{p}<0,001)$. Todas las patas fueron relevantes en la ordenación mediante componentes principales con sólo un componente relevante $(\lambda=3,7)$ que explica por si sólo el $92,2 \%$ de la variabilidad y con factores de carga de 0.98 para P2 y para P3, 0.97 para P1 y 0,90 para P4 (Figura 3).

El análisis discriminante mostro diferencias en los grupos con centroides -1.38 y 0.77 para machos y hembras respectivamente, mostrando un mayor peso de P1 y P2 en la discriminación de los grupos (FLD $=-0,72 \mathrm{P} 1$ $0,49 \mathrm{P} 2+0,09 \mathrm{P} 3+0,13 \mathrm{P} 4)$, con una probabilidad de buena clasificación de un $85,7 \%$ (Figura 4).

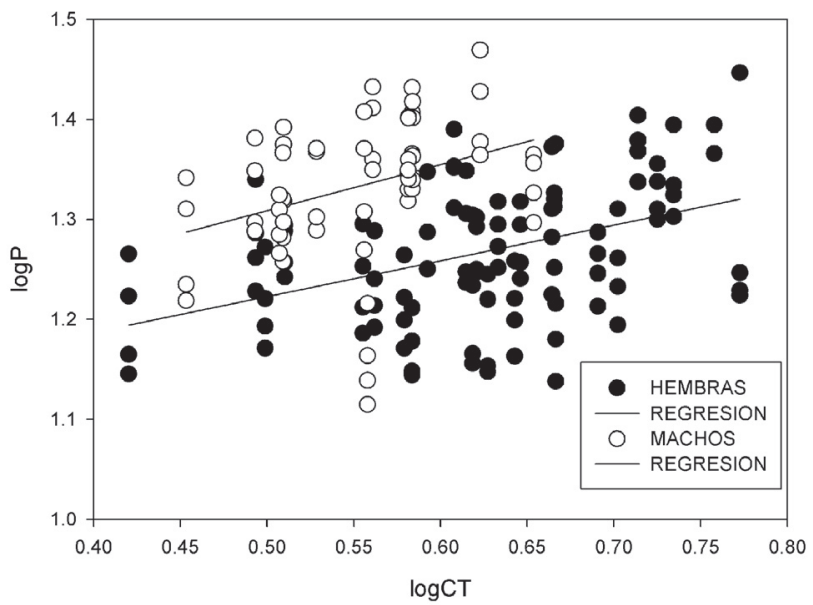

Figura 2. Alometría de las patas de L. laeta. Regresiones lineales entre el logaritmo de la longitud de las patas $(\log \mathrm{P})$ y el $\log$ aritmo de la longitud del cefalotórax $(\log \mathrm{CT})$ para hembras y machos de L. laeta. Para hembras $\log \mathrm{P}=1,04+0,36 \log \mathrm{CT}\left(\mathrm{R}^{2}=0,18 ; \mathrm{F}_{1,106}\right.$ $=24,1 ; \mathrm{p}<<0,001)$ y para machos $\log \mathrm{P}=1,08+0,46 \log \mathrm{CT}\left(\mathrm{R}^{2}=\right.$ 0,$\left.11 ; \mathrm{F}_{1,58}=7,4 ; \mathrm{p}<<0,001\right)$

FIGURE 2. Allometry of legs of L. laeta. Linear regressions between the logarithm of leg length $(\log \mathrm{P})$ and the logarithm of cephalotorax length $(\log \mathrm{CT})$ for females and males of L. laeta: $\log \mathrm{P}=1.04+0.36 \log \mathrm{CT}\left(\mathrm{R}^{2}=0.18 ; \mathrm{F}_{1,106}=24.1 ; \mathrm{p}<<0.001\right)$ for females and $\log \mathrm{P}=1.08+0.46 \log \mathrm{CT}\left(\mathrm{R}^{2}=0.11 ; \mathrm{F}_{1,58}=7.4 ; \mathrm{p}<<\right.$ 0.001 ) for males.

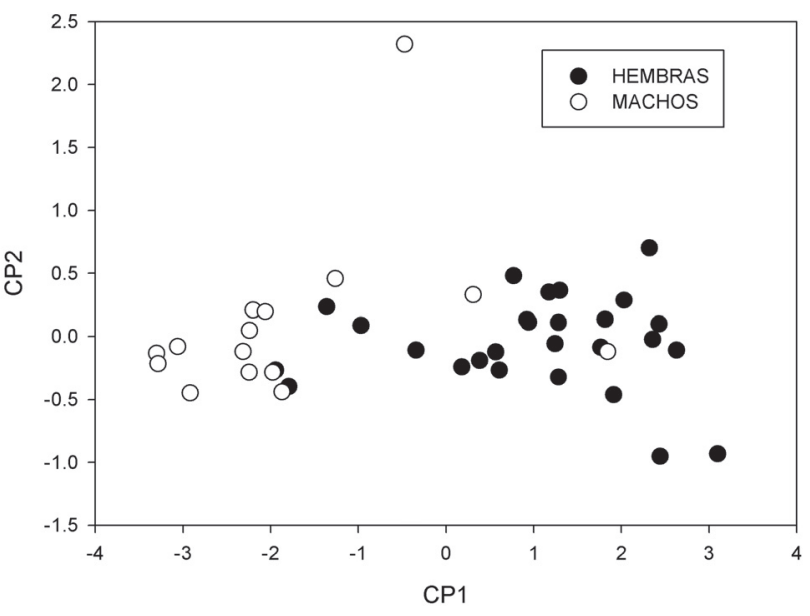

FIgURA 3. Ordenación con componentes principales de hembras y machos de L. laeta. CP1 y CP2 de hembras y machos de L. laeta, basados en las longitudes de las patas locomotoras. El primer componente explica el $92,2 \%$ y el segundo sólo el $6,2 \%$ de la variabilidad total.

FIgURE 3. Principal components ordination of females and males of L. laeta (CP1 and CP2) based on the lengtghs of locomotor legs. CP1 and CP2 explain $92.2 \%$ and $6.2 \%$ of the total variance, respectively.

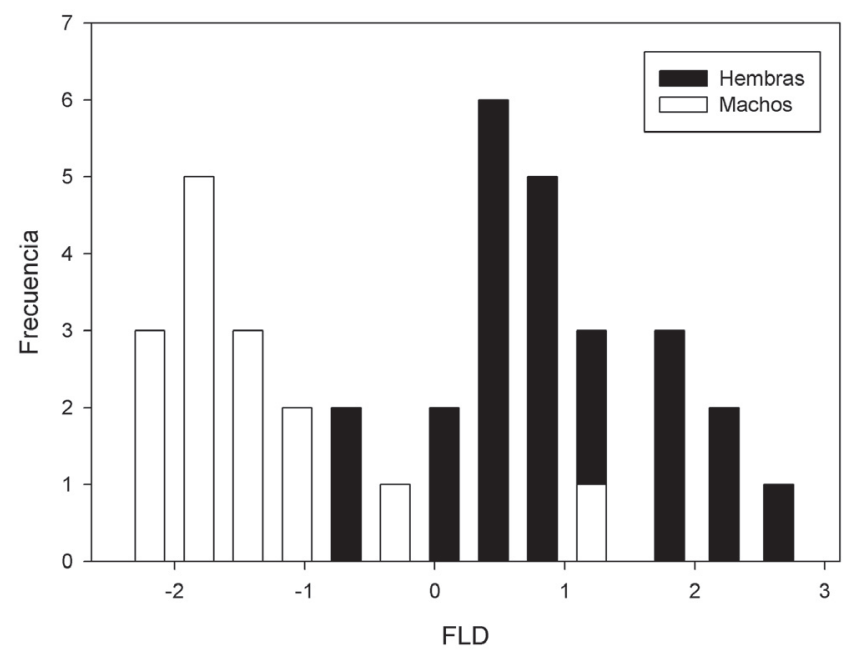

Figura 4. Discriminación de hembras y machos de L. laeta. Función lineal discriminante calculada (FLD)

Figure 4. Linear discriminant function (FLD) of females and males of L. laeta. 
Dimorfismo sexual y morfología funcional de L. laeta: Mauricio Canals et al.: MaURICIO CANALS et AL.

TABLA 1.- Dimensiones de las extremidades locomotoras de L. laeta $. \mathrm{T}=$ tarso, $\mathrm{MTT}=$ metatarso, $\mathrm{TB}=$ tibia, $\mathrm{PT}=$ patela, $\mathrm{F}=$ femur. $\mathrm{Los}$ números indican el orden de la extremidad.

TABLE 1.- Lengths of locomotor legs of $L$. laeta $. \mathrm{T}=$ tarsus, $\mathrm{MTT}=$ metatarsus, $\mathrm{TB}=$ tibia, $\mathrm{PT}=$ patella, $\mathrm{F}=$ femur. The numbers indicates the order of the legs.

\begin{tabular}{|c|c|c|c|c|}
\hline \multirow{2}{*}{$\begin{array}{c}\text { SEXO } \\
\text { LONGITUD }\end{array}$} & \multicolumn{2}{|c|}{ HEMBRAs } & \multicolumn{2}{|c|}{ MaCHOS } \\
\hline & Absoluta (mm) & RELATIVA & Absoluta (mm) & RELATIVA \\
\hline T1 & $1,65 \pm 0,26$ & $0,40 \pm 0,10$ & $1,88 \pm 0,35$ & $0,53 \pm 0,09$ \\
\hline MTT1 & $4,83 \pm 0,90$ & $1,15 \pm 0,25$ & $6,13 \pm 0,75$ & $1,70 \pm 0,14$ \\
\hline TB1 & $5,23 \pm 0,86$ & $1,25 \pm 0,25$ & $6,31 \pm 0,78$ & $1,79 \pm 0,19$ \\
\hline PT1 & $1,21 \pm 0,22$ & $0,29 \pm 0,06$ & $1,19 \pm 0,17$ & $0,32 \pm 0,05$ \\
\hline F1 & $5,00 \pm 0,98$ & $1,18 \pm 0,22$ & $5,66 \pm 0,67$ & $1,58 \pm 0,11$ \\
\hline PATA 1 & $17,92 \pm 2,71$ & $4,26 \pm 0,77$ & $21,15 \pm 2,01$ & $5,93 \pm 0,32$ \\
\hline $\mathbf{T 2}$ & $1,70 \pm 0,32$ & $0,40 \pm 0,08$ & $1,86 \pm 0,31$ & $0,52 \pm 0,09$ \\
\hline MTT2 & $5,18 \pm 0,85$ & $1,23 \pm 0,25$ & $6,93 \pm 1,02$ & $1,97 \pm 0,31$ \\
\hline TB2 & $5,44 \pm 0,85$ & $1,30 \pm 0,27$ & $6,72 \pm 0,89$ & $1,94 \pm 0,21$ \\
\hline PT2 & $1,20 \pm 0,22$ & $0,29 \pm 0,06$ & $1,20 \pm 0,13$ & $0,33 \pm 0,04$ \\
\hline F2 & $5,55 \pm 0,95$ & $1,32 \pm 0,26$ & $6,19 \pm 0,61$ & $1,74 \pm 0,15$ \\
\hline PATA 2 & $19,07 \pm 2,55$ & $4,55 \pm 0,81$ & $22,90 \pm 2,50$ & $6,50 \pm 0,69$ \\
\hline T3 & $1,55 \pm 0,33$ & $0,38 \pm 0,10$ & $1,58 \pm 0,18$ & $0,44 \pm 0,05$ \\
\hline MTT3 & $4,73 \pm 1,12$ & $1,12 \pm 0,28$ & $6,12 \pm 1,12$ & $1,77 \pm 0,26$ \\
\hline TB3 & $4,57 \pm 0,80$ & $1,08 \pm 0,19$ & $5,30 \pm 0,67$ & $1,53 \pm 0,12$ \\
\hline PT3 & $1,21 \pm 0,24$ & $0,29 \pm 0,05$ & $1,15 \pm 0,17$ & $0,32 \pm 0,05$ \\
\hline F3 & $5,11 \pm 0,88$ & $1,21 \pm 0,21$ & $5,65 \pm 0,75$ & $1,60 \pm 0,09$ \\
\hline PATA 3 & $17,12 \pm 2,62$ & $4,07 \pm 0,71$ & $19,82 \pm 2,35$ & $5,65 \pm 0,45$ \\
\hline T4 & $1,77 \pm 0,26$ & $0,43 \pm 0,07$ & $1,96 \pm 0,29$ & $0,51 \pm 0,11$ \\
\hline MTT4 & $6,16 \pm 1,34$ & $1,47 \pm 0,38$ & $7,83 \pm 1,31$ & $2,19 \pm 0,46$ \\
\hline TB4 & $5,75 \pm 0,88$ & $1,37 \pm 0,25$ & $6,71 \pm 0,91$ & $1,88 \pm 0,27$ \\
\hline PT4 & $1,33 \pm 0,29$ & $0,32 \pm 0,06$ & $1,21 \pm 0,14$ & $0,33 \pm 0,05$ \\
\hline F4 & $6,01 \pm 1,17$ & $1,42 \pm 0,26$ & $6,54 \pm 0,55$ & $1,75 \pm 0,43$ \\
\hline PATA 4 & $20,95 \pm 3,02$ & $4,98 \pm 0,90$ & $24,24 \pm 2,60$ & $6,66 \pm 1,20$ \\
\hline CEFALOTORAX & $4,30 \pm 0,81$ & & $3,78 \pm 0,87$ & \\
\hline $\mathbf{T}$ & $1,36 \pm 0,20$ & $0,41 \pm 0,08$ & $1,71 \pm 0,19$ & $0,47 \pm 0,05$ \\
\hline $\mathbf{T b}$ & $0,96 \pm 0,25$ & $0,29 \pm 0,09$ & $2,17 \pm 0,14$ & $0,64 \pm 0,04$ \\
\hline $\mathbf{P}$ & $0,54 \pm 0,14$ & $0,16 \pm 0,05$ & $0,98 \pm 0,09$ & $0,30 \pm 0,02$ \\
\hline $\mathbf{F}$ & $1,30 \pm 0,37$ & $0,39 \pm 0,13$ & $3,41 \pm 0,42$ & $1,04 \pm 0,07$ \\
\hline PALPO & $4,16 \pm 0,87$ & $1,25 \pm 0,33$ & $8,28 \pm 0,71$ & $2,44 \pm 1,21$ \\
\hline
\end{tabular}

\section{DISCUSIÓN}

Todos los ejemplares observados se ajustan a las descripciones de L. laeta realizadas por Gertsch (1967) y Gertsch \& Ennik (1983). El clípeo de hembras y machos corresponde a 3 veces el diámetro mayor de los ojos medianos en las hembras y en los machos más corto, aproximadamente 2,3 veces el diámetro. La razón entre el ancho y largo de la tibia del palpo de los machos fue $0,34 \mathrm{y}$ es consistente con la relación $1 / 3$ propuesta para esta especie (Gertsch 1967, 1983). La fórmula de patas en machos y hembras fue 4-2-1-3 lo que también es consistente con las descripciones previas, aunque desde el punto de vista estadístico en este estudio las diferencias de tamaño entre la pata 1 y 3 no alcanzaron significación.

El dimorfismo sexual es común en arañas (Moya-Laraño et al. 2002; Brandt \& Andrade 2007a; Moya-Laraño et al. 
2009; Foelix 2011). En L. laeta el dimorfismo sexual de las extremidades locomotoras fue claro, pero no extremo como en algunas arañas de telas orbitales de la familia Theridiidae los machos tienen el $1 \%$ de la masa de la hembra. Este resultado es consistente con lo reportado para otras arañas del suelo (Enders 1976; Prenter et al. 1999; Gasnier et al. 2002; Framenau 2005), pero menor que lo reportado para arañas tejedoras de telas (Hormiga et al. 1995; 2000).

Tanto machos como hembras mostraron una alometría negativa de las extremidades, es decir las arañas con tamaño mayor de cefalotórax tienen patas proporcionalmente más cortas que aquellas con cefalotórax más cortos, lo cual puede ser explicado por causas biomecánicas ya que la masa incrementa con el cubo de la longitud y la resistencia con el cuadrado de ésta (McMahon 1983). Por otra parte la alometría positiva se encuentra asociada a los órganos que se encuentran bajo selección sexual (Eberhard et al. 2000), lo que no es evidente en las extremidades locomotoras.

Las diferencias en los órganos locomotores entre los sexos puede ser favorecido por selección de la conducta de búsqueda del macho o por selección sobre los movimientos de la hembra durante la oviposición o el forrajeo (Framenau \& Hebets 2007). Sin embargo estas diferencias pueden no estar relacionadas con ventajas en la locomoción. Por ejemplo, las patas largas de los machos han sido atribuidas a competencia directa entre machos en los Gerridae (Tseng \& Rowe 1999) y escarabajos megalopodinos (Eberhard \& Marin 1996), al despliegue durante el cortejo en arañas lobo (Lycosidae) (Kronestedt 1990; Framenau \& Hebets 2007) y a la reducción del riesgo de canibalismo sexual en algunas arañas de tela (Elgar et al. 1990).

Sin embargo el patrón de dimorfismo sexual que encontramos ha sido reportado en otras arañas errantes del suelo, incluyendo Migalomorfas (Calderon et al. 1990; Gasnier 2002; Framenau 2005; Grossi et al. 2015). Por ejemplo, Gasnier (2002) reportó que los machos tienen mayor área que las hembras ya que tienen patas proporcionalmente mayores que las hembras en Phoneutria spp. y Ctenus spp. (Ctenidae), y Framenau (2005) reportó que en la araña lobo Venatrix lapidosa (McKay, 1974) que tiene hembras sedentarias, los machos tienen patas comparativamente mas largas que las hembras, mientras que en Artoria sp. cuyas hembras son errantes, las patas de las hembras y machos son proporcionalmente iguales, sugiriendo que la longitud de las patas está efectivamente asociada a la actividad locomotora, proporcionando fuerte evidencia que la elongación de las patas en los machos se encuentra asociada a la competencia indirecta de los machos por apareamiento.

Desde un punto de vista energético los machos con patas largas pueden incrementar su eficiencia locomotora
(Ghiseling 1974; Grossi \& Canals 2015). Las patas largas están relacionadas con mayor velocidad, lo cual puede ser determinante en las oportunidades de copular. Como una consecuencia de una baja interacción macho-macho en las arañas errantes con baja densidad poblacional, una alta velocidad, con bajos costo energético y costo de transporte puede ser favorecido por la selección natural. La mecánica del péndulo muestra las ventajas de las patas largas en arañas y su relación con la velocidad, especialmente en arañas que trepan o que cuelgan de telas (Foelmer \& Moya-Larraño 2007). El tamaño corporal pequeño está relacionado con bajo costo de transporte (Grossi \& Canals 2015) por lo que compensado por patas largas debería ser la morfología esperada para los machos más activos y móviles, lo que es consistente con los resultados encontrados en $L$. laeta.

\section{AGRADECIMIENTOS}

Los autores agradecen a la Dra Viviane Jeréz por estimular el desarrollo de este trabajo y a los Dres Jorge Artigas y Mario Elgueta por facilitar los ejemplares para su revisión en los Museos.

\section{BIBLIOGRAFIA}

Alfaro, C., Veloso, C., Torres-Contreras, H., Solís, R. \& Canals, M. 2013. Thermal niche overlap of the spider of the nook Loxosceles laeta (Araneae; Sicariidae) and its possible predator, the tiger spider Scytodes globula (Scytodidae). Journal of Thermal Biology 38:502-507.

Anderson, M. 1994. Sexual Selection. Princeton University Press, Princeton, New Jersey.

Brandt, Y. \& Andrade, M.c.B. 2007. Testing the gravity hypothesis of sexual size dimorphism: are small males faster climbers? Functional Ecology 21:379-385.

Calderon, R., Garrido, M. \& Pinto, C. 1990. Etapas del crecimiento de Acanthognatus franckii Karsch, 1880 (Araneae: Nemesidae). Revista Chilena de Entomología 18:19-24.

Canals, M., Alfaro, C., Veloso, C., Torres-Contreras, H. \& Solís, R. 2013. Tolerancia a la desecación y sobreposición del nicho térmico entre la araña del rincón Loxosceles laeta y un posible control biológico, la araña tigre Scytodes globula. Revista Ibero-Latinoamericana de Parasitología 72:60-74.

Canals, M., Arriagada, N. \& Solís, R. 2015. Interactions between the Chilean recluse spider and an araneophagic spitting spider. Journal of Medical Entomology 52:109-116.

Canals, M., Casanueva, M.e. \& Aguilera, M. 2004. Cuales son las especies de arañas peligrosas en Chile? Revista Médica de Chile 132:773-776.

Canals, M. \& Solís, R. 2013. Is the tiger spider Scytodes globula an effective predator of the brown recluse spider Loxosceles laeta? Revista Médica de Chile 141:805-807.

Canals, M. \& Solís, R. 2014. Desarrollo de cohortes y parámetros 
poblacionales de la araña del rincón Loxosceles laeta. Revista Chilena de Infectología 31:547-554.

Eberhard, W.G. \& Marin, M.C. 1996. Sexual behavior and the enlarged hind legs of male Megalopus armatus (Coleoptera, Chrysomelidae). Journal of Kansas Entomological Society 69:1-8.

Eberhard, W.G., García,C.J.M. \& Lobo, J. 2000. Size-specific defense structures in a horned weevil confirm a classic battle plan: avoid fights with larger opponent. Proceedings of the Royal Society of London B 267:1129-1134.

Elgar, M.A., Ghaffar, N. \& Read, A.F. 1990. Sexual dimorphism in leg length among orb-weaving spiders: a possible role for sexual cannibalism. Journal of Zoology London 222:455-470.

Elgar, M.A. \& FAHEY, B.F. 1996. Sexual cannibalism, competition, and size dimorphism in the orb-weaving spider Nephila plumipes Latreille (Araneae: Araneoidea). Behavioral Ecology 7:195-198.

EMLEn, D.J. 2008. The evolutionof animal weapons. Annual Review of Ecology Evolution and Systematics 39:387413.

ENDERS, F. 1976. Clutch size related to hunting manner of spider species. Annals of the Entomological Society of America 69:991-998.

FAIRBAiRn, D. J. 1997. Allometry for sexual size dimorphism: Pattern and Process in the Coevolution of Body Size in Males and Females. Annual Review of Ecology and Systematics 28:659-687.

FAIRBAIRN, D.J. 2013. Odd Couples. Extraordinary Differences between the Sexes in the Animal Kingdom. Princeton University Press, Princeton and Oxford.

Foellmer, M.W. \& Moya-Larraño, J. 2007. Sexual size dimorphism in spiders: pattern and processes. In: Sex, Size and Gender roles: Evolutionary studies of sexual size dimorphism (Eds. Fairbairn, D.J., Blanckenhorn, W.U. \& Székely, T.), pp 71-81. Oxford.

Foelix, R.F. 2011. Biology of Spiders. Oxford University Press, New York

Framenau, V.W. 2005. Gender specific differences in activity and home range reflect morphological dimorphism in wolf spiders (Araneae, Lycosidae). Journal of Arachnology 33:334-346.

Framenau, V., Reich, M. \& Plachter, H. 1996. Zum Wanderverhalten und zur Nahrungso"kologie von Arctosa cinerea (Fabricius, 1777) (Araneae: Lycosidae) in einer alpinen Wildflusslandschaft. Verhandlungen der Gesellschaft fur Oekologie 26:369-376.

Framenau, V.W. \& Hebets, E.A. 2007. A review of leg ornamentation in male wolf spiders, with the description of a new species from australia, Artoria schizocoides (Araneae, Lycosidae). Journal of Arachnology 35:89-101.

Galiano, M.E. 1967. Ciclo biologico e desarollo de Loxosceles laeta (Nicolet, 1849). Acta Zoologica Lilloana 23:431464.

Galiano, M.e. \& Hall, M. 1973. Datos adicionales sobre el ciclo vital de Loxosceles laeta (Nicolet) (Araneae). Physis $32: 277-288$

Gasnier, T.R., De Azevedo, C.S., TORRES-SANCHEZ, M.P. \& HOFER, H. 2002. Adult size of eight hunting spider species in central Amazonia: temporal variations and sexual dimorphism. Journal of Arachnology 30:146-154.

Gertsch, W.J. 1958. The spider family Plectreuridae. American Museum Novitates 1920:1-53.

Gertsch,W.J. 1967. The spider genus Loxosceles in South America (Araneae, Scytodidae). Bulletin of the American Museum of Natural History 136:117-174.

Gertsch, W.J. \& EnNIK, F. 1983. The spider genus Loxosceles in North America, Central America and the West Indies (Araneae, Loxoscelidae). Bulletin of the American Museum of Natural History 175:264-360.

GHISELIN, M.T. 1974. The economy of nature and the evolution of sex. University of California Press, Berkeley.

Grossi, B. \& Canals, M. 2015. Energetics, scaling and sexual size dimorphism of spiders. Acta Biotheoretica 63:71-81.

Grossi, B., Veloso, C., Taucare-Rios, A. \& Canals, M. 2016. Allometry of locomotor organs and sexual size dimorphism in the mygalomorph spider Grammostola rosea (Walckenaer, 1837) (Araneae, Theraphosidae). Journal of Arachnology 44(1):99-102.

Grossi, B., Solis, R., Veloso, C. \& Canals M. 2016. Consequences of sexual size dimorphism on energetics and locomotor performance of Grammostola rosea (Araneae; Teraphosidae). Physiological Entomology 41:281-288.

Hallander, H. 1967. Range and movements of the wolf spiders Pardosa chelata (O. F. Muller) and P. pullata (Clerck). Oikos 18:360-364.

HEad, G. 1995. Selection on fecundity and variation in the degree of sexual size dimorphism among spider species (class Araneae). Evolution 49:776-781.

Hormiga, G., Eberhard, W.G. \& Coddington, J.A. 1995. Webconstruction behavior in Australian Phonognatha and the phylogeny of nephiline and tetragnathid spiders (Araneae: Tetragnathidae). Australian Journal of Zoology 43:313364.

Hormiga, G., Scharff, N. \& Coddington, J.A. 2000. The phylogentic basis of sexual size dimorphism in orbweaving spiders (Araneae: Orbiculariae). Systematic Biology 49(3):435-462.

Kronestedt, T. 1990. Separation of two species standing as Alopecosa aculeata (Clerck) by morphological, behavioural and ecological characters, with remarks on related species in the pulverulenta group. Zoologica Scripta 19:203-225.

Kruger, O. 2005. The evolution of reversed sexual size dimorphism in hawks, falcons and owls: a comparative study. Evolutionary Ecology 19:467-486.

Lucas H. 1834. Description of Scytodes rufipes Lucas. En GuerinMeneville FE. Magasin Zoologie Paris 4:8, 2.

Mcmahon, T.A. 1983. On size and life. Scientific American Library. New York.

Montgomery, T.H. 1910. The significance of the courtship and secondary sexual characters of araneads. American Naturalist 44:151-177.

Malmgren, J.C. \& Thollesson, M. 2001. Sexual size and shape dimorphism in two species of newts, Triturus cristatus and T. vulgaris (Caudata: Salamandridae). Journal of Zoology London 249:127-136.

Morse, D.H. 2014. The relation of size to climbing, linecrossing and running performances of male crab spiders. Evolutionary Ecology 28:23-36. 
Moya-Laraño, J., Halaj, J. \& Wise, D.H. 2002. Climbing to reach females: Romeo should be small. Evolution 56: 420-425.

Moya-Laraño, J., Vinkovic, D., Allard, C.M. \& Foellmer, M.W. 2009. Optimal climbing speed explains the evolution of extreme sexual size dimorphism in spiders. Journal of Evolutionary Biology 22:954-963.

Nicolet, H. 1849. Aracnidos. En GAY C. Historia física y política de Chile. Zoologica 3:319-543.

Ohtsuka, S. \& Huys, R. 2001. Sexual dimorphismo in calanoid copepods: morphology and function. Hydrobiologia 453:441-466.

Peters, W.L. \& Campbell, I.C. 1991. Ephemeroptera (Mayflies). Pp. 279-293. In: The Insects of Australia. A Textbook for Students and Research Workers (CSIRO, ed.). Melbourne University Press, Carlton.

Platnick, N.I. 2004. The world spider catalog. The American Museum of Natural History, Merrett P \& HD Cameron Eds, New York.

Prenter, J., Elwood, R.W. \& Montgomery, W.I. 1998. No association between sexual size dimorphism and life histories in spiders. Proceedings of the Royal Society of London, B 265:57-62.

Prenter, J., Elwood, R.W. \& Montgomery, W.I. 1999. Sexual size dimorphism and reproductive investment by female spiders: a comparative analysis. Evolution 53 (6): 19871994.

Prenter, J., Montgomery, W.I. \& Elwood, R.W. 1997. Sexual dimorphism in northern temperate spiders: implications for the differential mortality model. Journal of Zoology, London 243:341-349.

Prenter, J., Perez-Staples, D. \& Taylor, PW. 2010a. Functional relations between locomotor performance traits in spider and implications for evolutionary hypotheses. BMC Research Notes. 3:306-311.

Prenter, J., Perez-Staples, D. \& Taylor, P.W. 2010b. The effects of morphology and substrate diameter on climbing and locomotor performance in male spiders. Functional Ecology 24:400-408.

ReISS, M.J. 1989. The Allometry of Growth and Reproduction. Cambridge University

Richter, C.J., Den Hollander, J. \& Vlijm, L. 1971. Differences in breeding and motility between Pardosa pullata (Clerck) and Pardosa prativaga (L. Koch), (Lycosidae, Araneae) in relation to habitat. Oecologia 6:318-327.

Sánchez-Quiróz, C., Arévalo, E. \& Barrantes G. 2012. Static allometry and sexual size dimorphism in Centruroides margaritatus (Scorpiones: Buthidae). Journal of Arachnology 40:338-344.

SAnTos, A.J. 2007. Evolucao do dimorfismo sexual de tamanho em aranhas. In Ecologia e Comportamento de Aranhas (Eds. Gonzaga, M.O., Santos, A.J. \& Japyassú, H.F.), pp. 137165. Editora Interciencia, Rio de Janeiro.

Schenone, H. 2003. Cuadros tóxicos producidos por mordedura de araña en Chile: latrodectismo y loxoscelismo. Revista Médica de Chile 131:437-444.

Stern, D.L. \& Emlen, D.J. 1999. The developmental basis for allometry in insects. Development 126:1091-1101.

Taucare-Ríos, A. 2011. Loxosceles surca (Gertsch, 1967) (Araneae: Sicariidae) en el norte de Chile. Boletín de Biodiversidad de Chile 5:45-49.

Thompson, D.W. 1961. On growth and form.Cambridge University Press, Cambridge.

Thornhill, R. \& Alcock, J. 1983. The Evolution of Insect Mating Systems. Cambridge University Press, Cambridge.

Tseng, M. \& Rowe, L. 1999. Sexual dimorphism and allometry in the giant water strider Gigantometra gigas. Canadian Journal of Zoology 77:923-929.

Vollrath, F. \& Parker, G.A. 1992. Sexual dimorphism and distorted sex ratios in spiders. Nature 360:156-159.

Recibido: 01.03.16

Aceptado: 23.08.16 